\title{
Irradiation history and atmospheric ablation of meteorites: Results from cosmic ray track studies*
}

\author{
J N GOSWAMI \\ Physical Research Laboratory, Ahmedabad 380 009, India
}

\begin{abstract}
The dominant component of nuclear tracks observed in meteoritic minerals poor in uranium is produced by cosmic ray very heavy (vH: $Z>20$ ) nuclei. Studies of cosmic ray tracks and other cosmogenic effects in meteorites give us information on the irradiation history of these meteorites and enable us to estimate the extent of ablation during their atmospheric transit, and hence their pre-atmospheric masses. In a specific type of meteorite, known as gas-rich meteorite, one finds individual grains and xenoliths that have received solar flare and galactic cosmic ray irradiation prior to the formation of these meteorites. Detailed studies of these exotic components give insight into the accretionary processes occurring in the early history of the solar system. Some of the important results obtained from such studies and their implications to meteoritics are summarized.
\end{abstract}

Keywords. Tracks; cosmic ray; meteorite; ablation; solar flares; irradiation history; exposure age; accretionary processes.

\section{Introduction}

The nuclear tracks observed in silicate grains from extraterrestrial samples (e.g. lunar samples and meteorites) are produced by one or more of the following components:

(i) stopping solar flare and galactic cosmic ray (GCR) very heavy (VH: $Z>20$ ) nuclei,

(ii) spontaneous fission of ${ }^{288} \mathrm{U}$ and, in some cases ${ }^{244} \mathrm{Pu}$,

(iii) induced fission of $\mathrm{U}$ and $\mathrm{Th}$, and

(iv) nuclear reaction-induced spallation recoils.

In the case of uranium-poor silicate grains in meteorites and lunar samples, the dominant source of tracks is the stopping solar flare and GCR heavy nuclei. The spallation recoil tracks have extremely short range $(<1 \mu)$ and are relatively unimportant except for lunar samples and meteorites with very long exposure ages. This can also be distinguished from the VH nuclei tracks, which have recordable track lengths of $\geqslant 10 \mu$ in common silicate grains. The low-energy (1-100 MeV/amu) solar flare particles have a maximum range of about a millimetre equivalent of silicate material, and as such solar flare vH nuclei tracks can be seen only in soil grains and rocks exposed on the lunar surface. In the case of meteorites, the solar flare track records are completely lost due to the atmospheric ablation processes which remove, on an average, a couple of centimetres of the surfacial material. A specific meteorite type, the gas-rich meteorite, is however, an exception, and one can find solar flare track records in silicate grains extracted from the interior of these meteorites, indicating that these grains were exposed to low-energy solar flare radiations prior to their incorporation into these meteorites. The nuclear tracks observed in most of the meteo-

\footnotetext{
*Paper presented at the second national seminar-cum-workshop on the use and application of solid state nuclear track detectors held at Physical Research Laboratory, Ahmedabad, India, during 24-26 February 1981.
} 
ritic silicate grains are thus produced primarily by vH nuclei in galactic cosmic rays. A detailed study of the track records in meteorites along with studies of other cosmogenic records (e.g. noble gas and radionuclide concentrations) gives information on: (i) the exposure and irradiation history of meteorites in space and (ii) the extent of atmospheric ablation and hence their pre-atmospheric masses. The study of individual components in gas-rich meteorites, that have received pre-compaction solar flare irradiation, on the other hand, offers us the unique opportunity to investigate possible changes in solar activity in the past and also to understand the accretionary processes occurring in the early history of the solar system. The present paper deals with some of the important aspects of the above studies and the present status of our understanding in these fields.

\section{Irradiation history of meteorites}

The cosmic ray exposure history of the meteorites, in general, is considered to be a single stage process involving removal of these objects from their parent bodies at some time in the past, and their exposure in space as small objects before being captured by the earth in recent times. It is generally assumed that while they were present in their parent bodies (the asteroids being the most probable parent bodies of meteorites) the meteorites were well shielded from cosmic rays. Such an assumption is justified since the mean attenuation length of various cosmogenic interactions varies from less than a micron (for solar wind interaction) to, at best, a couple of metres (for production of stable isotopes and radionuclides). There are, however, exceptions to the single stage exposure of meteorites, the gas-rich meteorites being one example. There are also other meteorites that have probably experienced multistage exposures, resulting from their presence in the nuclear active zone in the parent bodies, and collisional fragmentation in space after being ejected as small objects from the parent bodies. Exposure histories of each of the above types of meteorites will be discussed separately.

\subsection{Meteorites with single-stage exposure history}

In meteorites with single-stage exposure history, the cosmogenic records observed in them (e.g. tracks, spallogenic stable- and radio-nuclides, etc.) can be interpreted in terms of two parameters: (i) the intensity of the galactic cosmic rays, and (ii) the cosmic ray exposure durations. Studies of cosmogenic effects in both lunar and meteorite samples have shown that the long-term average intensity of both the proton and heavy ion components in GCR, responsible for production of cosmogenic nuclides and nuclear tracks respectively, has essentially remained constant for the last few tens of millions years (Arnold et al 1961; Lal 1972; Walker 1975; Reedy et al 1981). We shall therefore assume a constancy in galactic cosmic ray intensity in the past in the discussion that follows.

Assuming a constancy in GCR intensity it is possible, in principle, to obtain the duration of cosmic ray exposure $T$ of a meteorite by studying the cosmogenic records in it. However, the process of atmospheric ablation, which results in the removal of variable amounts of near surface material in different meteorites, introduces an additional uncertainty regarding the shielding depths of the analysed samples within the 
original meteorite, and hence on the exposure age estimates. Also, because of the extremely depth-sensitive production rate of nuclear tracks, the track records in meteorites alone cannot be used to estimate the duration of cosmic ray exposure. The problem is less severe in the case of cosmogenic stable- and radio-nuclide studies, since their production rates as a function of depth do not vary too much, and one can use an average production rate, independent of the shielding depth of the analysed samples, to obtain approximate exposure ages of meteorites. Examples of typical production rate profiles for nuclear tracks and stable isotopes and radionuclides in spherical meteroites of different sizes are shown in figure 1 . One can immediately note the large difference in the production rate profiles for tracks and cosmogenic nuclides. An added advantage in the case of cosmogenic nuclides is the possibility of studying more than one radionuclide or more than one isotope of the same element in the case of stable-nuclides, which gives an additional clue for introducing shielding depth corrections to the exposure age estimates (Eberhardt et al 1966). Studies of exposure age distribution of meteorites, based on measurements of cosmic ray produced stable isotopes using mass-spectrometric techniques, have led to groupings in the exposure age distributions of different meteorite types (Wanke 1966; Crabb and Schultz 1981). These groupings have been attributed to discrete collisional events involving the parent body/bodies of the different meteorite types at different times in the past.

An accurate knowledge of the shielding depths of the analysed samples and the size of the original meteorites is however important for a better understanding of the exposure and irradiation history of individual meteorites, and particularly in the case of small meteorites where the production rates of cosmogenic nuclides vary signi-
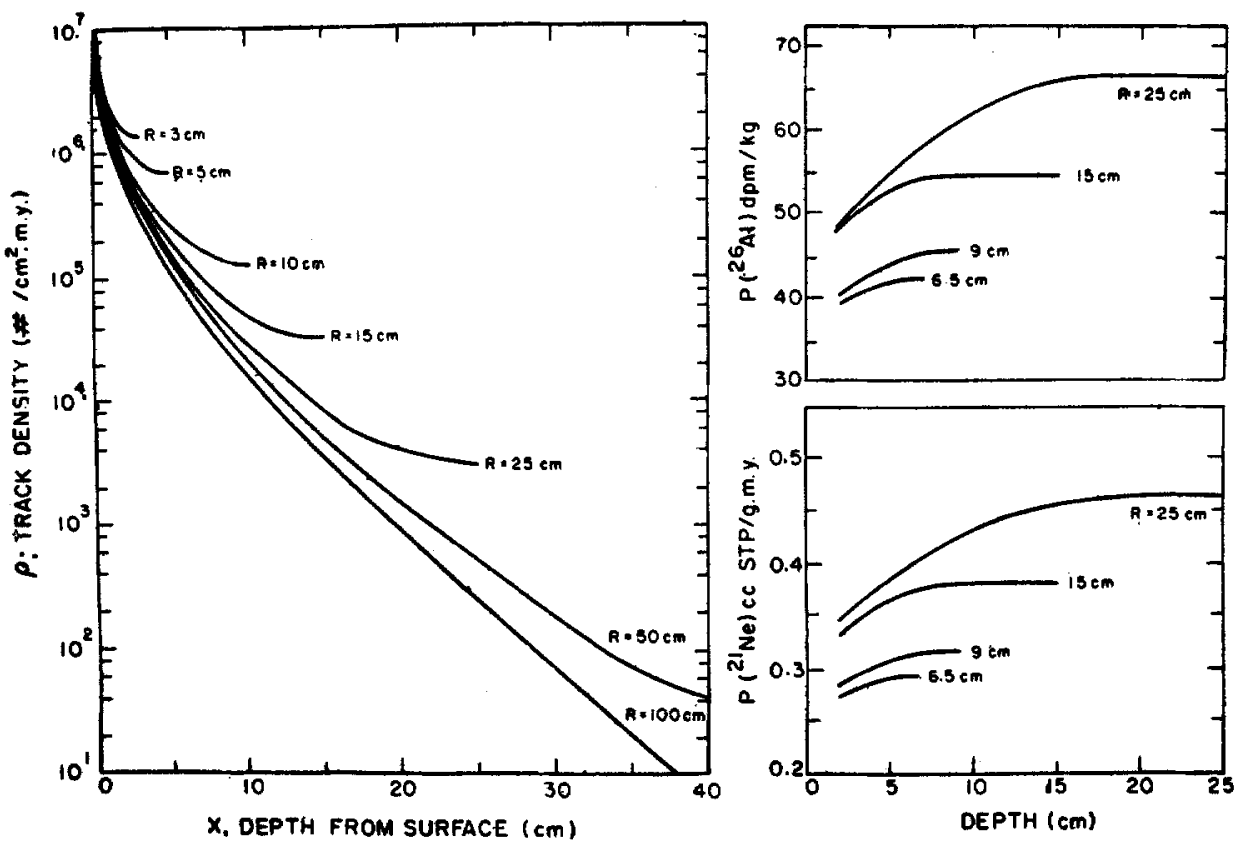

Figure 1. Production rate of cosmic ray produced tracks, radio-nuclide $\left({ }^{20} \mathrm{Al}\right)$ and stable isotope $\left({ }^{21} \mathrm{Ne}\right)$ in meteorites of chondritic composition. The $P\left({ }^{21} \mathrm{Ne}\right)$ values are in the unit of $10^{-8}$. 
ficantly at a given shielding depth for different sizes of meteorites (figure 1). It is therefore essential to understand the magnitude of atmospheric ablation of meteorites, and for this purpose, the study of nuclear track records would be ideal because of its extremely depth sensitive production rate.

\section{Atmospheric ablation of meteorites}

The magnitude of atmospheric ablation of meteoroids during their passage through the earth's atmosphere depends on various parameters e.g. velocity, angle of entry, mass, density, shape etc. of the meteoroids. Analytical treatment of this problem to determine the dependence of meteoroid ablation on the above parameters has been carried out by various authors (Opik 1958; Baldwin and Sheaffer 1971; Revelle 1978). The discussion in this paper will be confined to studies based on the nuclear track method.

The basic principle involved in determining the extent of atmospheric ablation of meteoroids using the nuclear track method has been discussed by Fleischer et al (1967) and Bhattacharya et al (1973). The first step is to obtain the values of shielding depths (depth of the sampling point from the original (pre-atmospheric) meteoroid surface) for different sampling points within the recovered (post-atmospheric) meteorite. This involves determination of cosmic ray track densities in silicate grains taken from the sampling locations and evaluation of the rate of production of tracks, TPM (no. of $t$ racks per $m . y$.), using the information on cosmic ray exposure age of meteorites obtained from stable isotope and/or radio-nuclide studies. Since the track production rate at a given depth $X$ is not very sensitive to the size of the meteorite (see figure 1), one can easily evaluate the shielding depth $\Delta X$ of any given sampling point. Bhattacharya et al (1973) have generated a set of graphs, using the track production profile shown in figure 1 , which directly gives the shielding depth $\Delta X$ as a function of both TPM and effective recovered radius of the meteorites and can be used conveniently to obtain $\Delta X$ values. In generating these $\Delta X$ vs TPM graphs, Bhattacharya et al (1973) assumed that the ablation is symmetric. However, the error introduced in the estimate of shielding depths, because of this assumption, is not serious since for $a \pm 100 \%$ error-estimate in the actual radius of the meteorite, the error in shielding depth is less than $\mp 10 \%$.

The methods of estimating pre-atmospheric size and radius of the meteorites for different ablation characteristics, based on shielding depth values obtained from nuclear track studies, have been discussed in detail by Gupta and Lal (1978). They have considered cases involving both symmetric and asymmetric ablation, and also survival of meteoroids as single or multiple fragments. The important results obtained in the work of Gupta and Lal (1978) are summarized below:

(i) In the case of a single surviving fragment, it is possible to accurately reconstruct the pre-atmospheric size and shape of the meteorites if the centre of the pre-atmospheric body is contained within the recovered fragment.

(ii) If the centre of the original meteorite is not contained within the recovered body, reconstruction of pre-atmospheric size is not possible in a straight-forward manner. However if a large data set for shielding depths at various points of the recovered fragment can be obtained, one can estimate the pre- 
atmospheric size by comparing the expected and observed probability distributions of shielding depths for meteorites of different sizes which have suffered different degrees of ablation.

(iii) In the case of meteorite fall involving multiple fragments one can estimate approximate pre-atmospheric size from the lowest track density value, obtained after extensive analysis of a large number of samples, assuming that the lowest density sample represents the centre of the original meteorite. Another approach is to estimate mass-loss for different track density intervals based on the observed and expected recovered masses for different track density intervals.

Earliest studies of atmospheric ablation of meteorites using the nuclear track method were carried out by Fleischer et al (1967), and more extensive studies were carried out to determine pre-atmospheric sizes of different meteorites by Price $e t$ al (1967), Cantelaube et al 1969, Lal et al (1969), Lorin and Pellas (1975), Bull and Durrani (1976), Goswami et al (1978) and Bagolia et al (1977, 1978, 1980). The ablation phenomena in more than 150 meteorites have been studied recently by Bhandari et al (1980). Following the methods of Bhattacharya et al (1973) and Gupta and Lal (1978), Bhandari et al (1980) have obtained 'good', 'model' and 'limit' estimate of pre-atmospheric masses for a large number of meteorites, classified into four sub-groups, based on the frequency distribution of the shielding depths of samples analysed from each meteorite. 'Good' estimate for pre-atmospheric masses could be obtained only for about $20 \%$ of the analysed meteorites, since extensive sampling is necessary to obtain such estimates. They have found that the mass-ablation estimate ranges between 25 to $99.9 \%$ of the original mass, with a weighted mean of $\sim 85 \%$. The frequency distribution of pre-atmospheric masses $\left(M_{0}\right)$ was found to follow a distribution of the type $d N \propto M_{0}^{-1} d M_{0}$. There is also a suggestion in the data that a relation between the mean mass-ablated and the atmospheric velocity of the meteorite exists in the velocity range 14 to $20 \mathrm{~km} \mathrm{sec}^{-1}$.

The frequency distribution of minimum shielding depths for different meteorites grouped as a function of percentage mass-ablation, obtained by Bhandari et al (1980), is shown in figure 2. The small values of minimum shielding depth, even in the cases of high ablation mass loss, indicate multiple fragmentation in the initial part of the trajectory since the outer regions, corresponding to low $\Delta X$ values, will be ablated away in the absence of fragmentation. The results obtained from nuclear track studies of the Dhajala shower (Bagolia et al 1977) illustrates such a phenomenon. The frequency distribution of the pre-atmospheric masses for the 'good' estimate cases is shown in figure 3. The roughly equal populations in the mass interval, $M_{0}=10-1000 \mathrm{~kg}$, indicate a power law distribution of the type $d N \propto M_{0}^{-1} d M_{0}$, as noted earlier. Such a distribution is distinctively flatter than the estimated index of about -2 for meteoroids in the mass-range $1-10^{8} \mathrm{~g}$ (Gault et al 1974), and of about -1.6 for meteoroids in the mass range $10^{3}-10^{7} \mathrm{~g}$ (McCrosky 1968). However, as Bhandari et al (1980) have noted, the lower efficiency in recovering small meteorites introduces a definite bias in the track based data towards meteorites of larger sizes.

The estimation of shielding depth by the track method has also been proved to be extremely useful in obtaining accurate production rates of cosmogenic nuclides in meteorites of various sizes. A combined study of nuclear track and cosmogenic nuclides in several meteorites, carried out recently by Bhattacharya et al (1980), 


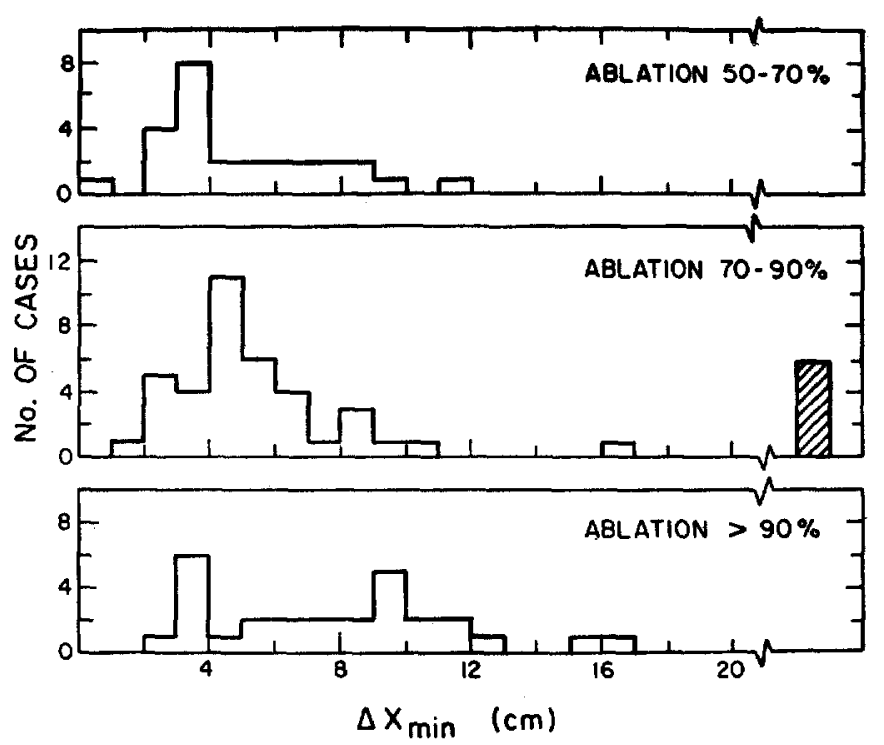

Figure 2. Frequency distribution of minimum shielding depth $\left(\Delta X_{\min }\right)$ values for meteorites in three different ablation intervals. The no. of cases for which $\Delta X_{\min }$ exceeds $20 \mathrm{~cm}$ are combined together (from Bhandari et al 1980).

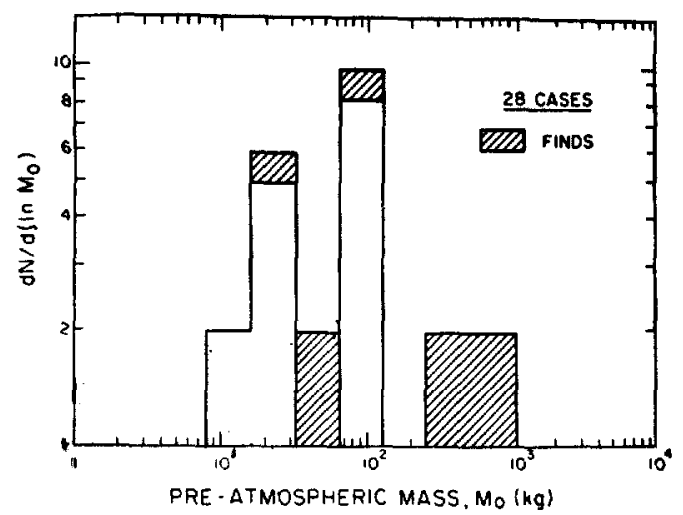

Figure 3. The number of meteorites in equal logarithmic mass interval is shown based on data for 28 meteorites for which reliable estimates of pre-atmospheric masses have been obtained using the nuclear track method (from Bhandari et al 1980).

led to the estimation of the parameter defining the hardness of energy spectra of cosmic ray primary and secondary nucleons at different depths within the meteorites of different sizes. This information is crucial in obtaining the production rates of cosmogenic nuclides as a function of depth for meteorites of different sizes.

The temperature gradient within a meteorite generated during the atmospheric ablation process also leads to partial annealing of tracks in the near surface (crusted) region of the recovered meteorite, and partial fading of low-temperature thermoluminescence. Studies of these effects coupled with laboratory data on track annealing and thermoluminescence fading characteristics can, therefore, give valuable insight into the atmospheric ablation process itself. Some work on these lines have recently been initiated (Jha and Lal 1981; Singhvi et al 1981). 


\section{Meteorites with multiple exposure histories}

In the preceding discussion on the atmospheric ablation of meteorites it has been assumed that the meteorites have had a simple (one-stage) cosmic ray exposure in space as small objects. However considering the fact that collisional events (both cratering and catastrophic disruption of large objects) play an important role in the generation of meteorites, one expects some of the meteorites to have multiple cosmic ray exposure histories, prior to their exposure in space as a small object in the recent past. Such pre-irradiation could take place if the meteorites resided within the nuclear active zone (the upper few metres) of the first or second generation parent objects from which they were finally removed during collisional events. MonteCarlo calculations of collision processes taking place among inner solar system objects (Turner 1979; Wetherill 1980) indicate that instances of multiple exposure of meteorites may be quite common. For example, in the case of meteorites with pre-atmospheric masses in the range $10-1000 \mathrm{~kg}$, the probability of multiple exposure could be as high as $50 \%$ (Wetherill 1980). This result, however, depends on the assumed values of certain parameters characterizing the number distribution of small-size objects in the inner solar system and the collisional processes involved, and thus may be somewhat uncertain. Nonetheless, an analysis of meteorites with multiple exposure history offers a unique opportunity of studying the finer details in the evolutionary processes of solar system objects.

Although gas-rich meteorites can be cited as a specific case of meteorites with multiple exposure history, pre-irradiation in this case primarily refers to short-duration solar wind and solar flare irradiation of a few percent of the constituent components of these meteorites. Further, the records of solar wind and solar flare irradiations can be easily detected using particle track and mass-spectrometric methods. However, in non-gas-rich meteorites, devoid of solar wind-solar flare irradiation records, it is rather difficult to pinpoint cases of multiple exposure history, since, in the absence of any a priori knowledge of probable multiple exposure, the records of any given cosmogenic products (e.g. track, spallogenic stable isotopes and radionuclides) could be explained by considering an 'effective' single stage exposure. A method has been suggested recently by Bhandari and Potdar (1981) to detect cases of meteorites with multiple exposure history from a combined analysis of nuclear track and cosmogenic noble gas data. Based on the calculation of neon isotopic ratio $\left({ }^{22} \mathrm{Ne} /{ }^{21} \mathrm{Ne}\right)$ in meteorites of different sizes, and comparing the results of these calculations with measured values of this ratio in meteorites, whose pre-atmospheric masses have been determined by the track method, Bhandari and Potdar (1981) concluded that for a shielding depth $<30 \mathrm{~cm}$, meteorites in all sizes show a (22/21) ratio of $\geqslant 1.08$. Thus meteorites with measured $(22 / 21)$ ratios of $<1.06$ indicate a deep seated $(>30 \mathrm{~cm})$ exposure in larger size parent bodies. Their approach, shown in figure 4 , is to study the relationship between cosmogenic neon isotopic ratio and the measured track production rate (assuming an 'effective' single stage exposure) and to identify meteorites having probable multiple exposures histories. They have reported that $\sim 20 \%$ of the meteorites for which data have been analysed so far could have had multiple cosmic ray exposure histories. A more elaborate discussion of this topic will be found in the accompanying paper by Bhandari (1981).

A very strong case for multiple cosmic ray exposure histories of a couple of Antarctic meteorites was presented by Imamura et al (1979), based on a combined analysis of 


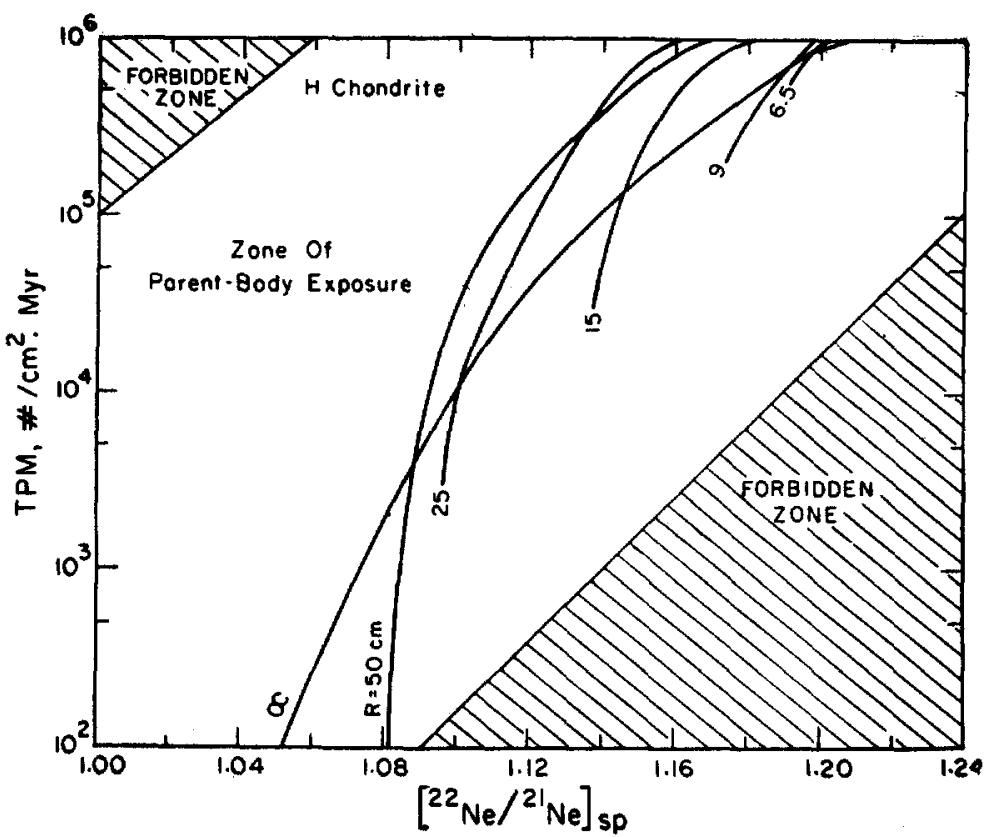

Figure 4. Correlation plot of cosmic ray produced neon isotopic ratio $\left({ }^{22} \mathrm{Ne} /{ }^{21} \mathrm{Ne}\right)_{s p}$ and track producition rate (TPM) for chondritic meteorites of different sizes and with a simple single stage cosmic ray exposure history. The hatched portion indicates forbidden zones. Data for meteorites with multiple exposure histories should deviate from the trend lines towards the upper left.

radionuclide and stable isotope data. Further work in this direction based on combined studies of nuclear track, noble gas and radionuclides is extremely important for a proper understanding of the collision-controlled evolutionary history of the small objects in the inner solar system.

\section{Irradiation and evolutionary history of the gas-rich meteorites}

The gas-rich meteorites can be considered as a special meteorite type having multiple exposure history. They contain individual components that have received solar-wind and solar flare irradiation prior to their compaction into the meteorite bodies. The irradiation records survived the compaction/brecciation processes leading to the formation of these meteorites, and can be easily detected using the nuclear track and mass spectrometric methods. In figure 5 are shown tracks due to solar flare heavy nuclei in grains from two gas-rich meteroites. The occurrence of such grains varies from $\sim 1$ to $20 \%$ among the grains present in these meteorites.

Gas-rich meteorites are present in varying proportion in different stony meteorites -both chondrites and achondrites. All the type 1 carbonaceous chondrites (cl), and $65 \%$ of the type-2 carbonaceous chondrites (CM) are gas-rich. Among other chondritic type the percentage abundance of gas-rich meteorites varies from zero (C3(0) chondrites) to $\sim 20 \%$. Among the achondrites, the aubrites and the howardites have gas-rich members. 


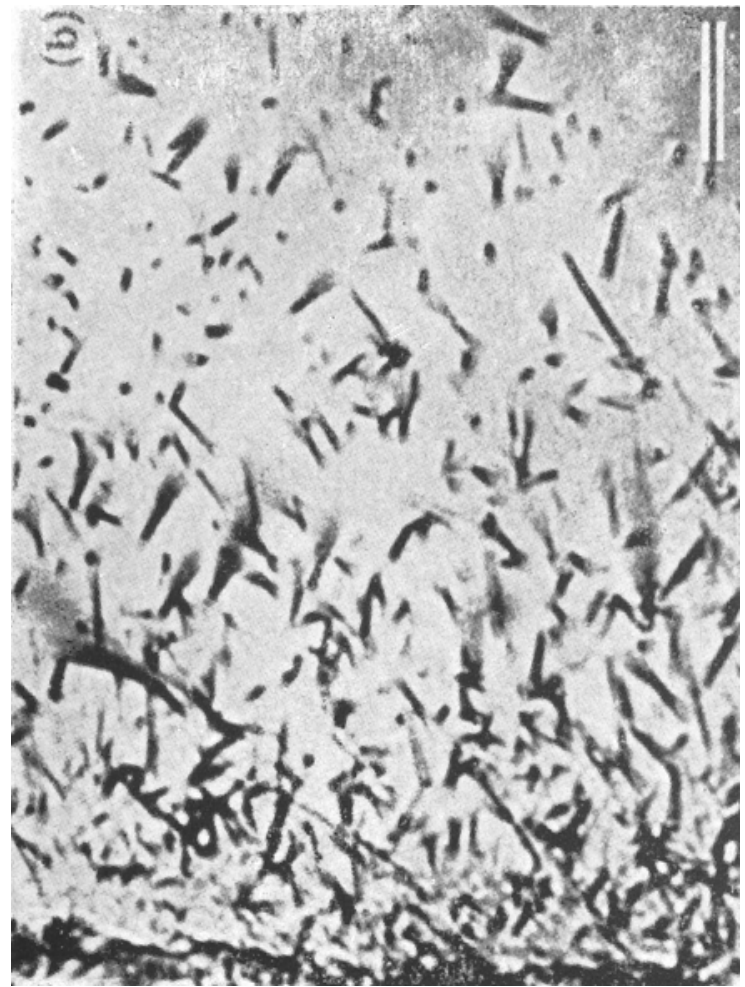

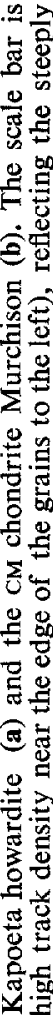

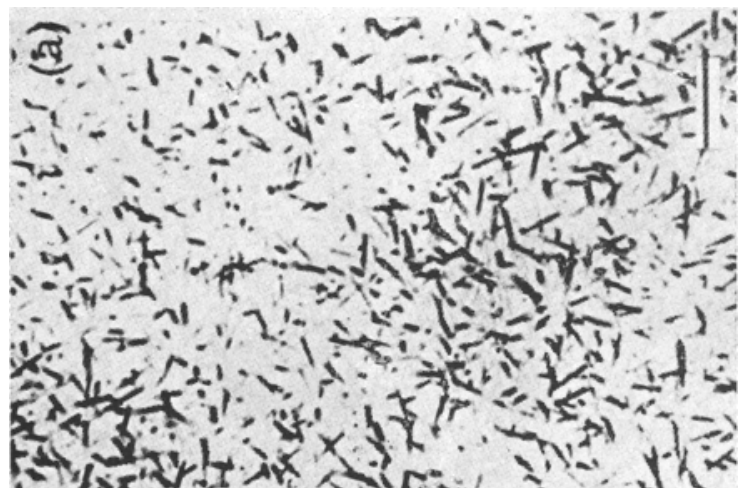
-

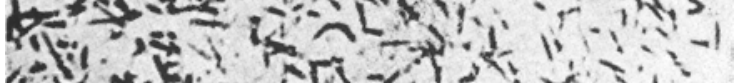

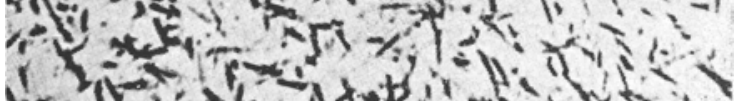
(n)

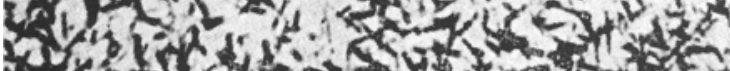
2. Civ'

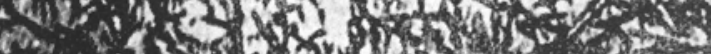

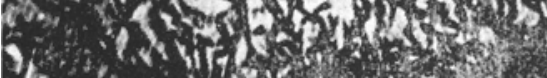


Since the solar wind and solar flare irradiation of individual components of gasrich meteorites, comprising both single grains and xenoliths (rock fragments), preceded the compaction of these meteorites, the study of the irradiation records in these components provide the unique opportunity for obtaining information on the solar activity at different epochs in the past. Although it is difficult to obtain information about the time of compaction of the gas-rich meteorites, the available data suggest that the compaction of the $\mathrm{CI}$ and CM chondrites took place more than 4 b.y. (billion years) ago (Macdougall and Kothari 1976) and it could be about 1.4 to 3.7 b.y. ago for other types of chondrites and achondrites (Schultz and Signer 1977; Rajan et al 1979). Information on the intensity and composition of solar flare particles during the early history of the sun, based on studies of gas-rich meteorites, has been summarized by Rajan (1974), Goswami et al (1980) and Crozaz (1980).

Apart from obtaining information about the activity of the ancient sun, studies of nuclear tracks and other cosmogenic effects in samples of gas-rich meteorites also provide valuable insight into the dynamical accretionary and evolutionary process of solar system objects. There is a general consensus at present that most of the gas-rich meteorites are regolithic* breccias from an asteroidal surface, and that the individual components of these meteorites received their solar flare and solar wind irradiation while residing in the asteroidal regolith. This scenario which was postulated quite some time back (Suess et al 1964) gained wider acceptance only after the analysis of lunar samples and the observed similarity in the solar wind-solar flare records of the gas-rich meteorites and the lunar soils and soil breccias, which are definitive regolith products, and some of which are gas-rich in character (Lal and Rajan 1969; Pellas et al 1969; Wilkening 1971; Pellas 1972; Poupeau and Berdot 1972; Rajan 1974; Poupeau et al 1974). Detailed comparison of solar flare irradiation records in lunar soil grains and track-rich grains in gas-rich meteorites and the observed anisotropy in the track irradiation pattern in the track rich grains ruled out the possibility that these grains were irradiated individually in free-space prior to their compaction into these meteorites (such an irradiation would have led to an isotropic track irradiation pattern). As the case for regolith evolution of gas-rich meteorites has been strengthened, attempts have been made to characterize the nature of the asteroidal regolith and the associated impact processes, and model the dynamical evolution of the asteroidal regolith (Anders 1975, 1978; Price et al 1975; Housen et al 1979; Langevin and Maurette 1980), based on our present understanding of processes taking place in the lunar regolith (Gault et al 1974; Arnold 1975; Langevin and Arnold 1977). The effect of several important parameters e.g. differences in gravity field and the flux and velocity of bombarding objects etc., in the lunar and asteroidal cases, has to be considered in these calculations. The results obtained indicate much higher values for the regolith thickness on asteroidal objects, extending upto several hundreds of metres, as opposed to about ten metres in the case of the moon. Although there seems to exist a qualitative agreement between the observed solar wind-solar flare irradiation features in gas-rich meteorites and those predicted on the basis of regolith evolution models, it must be noted here that in all the attempts made so far to explain the cosmogenic features seen in gas-rich meteorites, the pre-compaction exposure

*The term 'regolith' refers to the upper layers of dust and rocklets produced by impact processes on atmosphereless bodies of the solar system (e.g. moon, asteroids). The thickness of this layer depends on the physical characteristics of the bodies and the impacting objects, and can vary from a few metres to a few hundred metres. 
duration of individual components of these meteorites within the 'nuclear-active" region in the asteroidal regolith, has been considered as a free parameter or equated to the total cosmic ray exposure duration. Based on a re-evaluation of this problem Goswami and Lal (1979) postulated that the regolith irradiation scenario for gas-rich meteorites is not compatible at least in the case of the $\mathrm{CI}$ and $\mathrm{CM}$ (carbonaceous type 1 and 2) chondrites. They have based their argument on the fact that the pre-compaction irradiation durations, during which the individual components of the gasrich meteorites must have received their solar flare and solar wind irradiation, is extremely short $\left(<10^{5} \mathrm{yr}\right.$ ) in the case of $\mathrm{CI}$ and $\mathrm{CM}$ chondrites, and have shown that such short duration irradiation in the asteroidal regolith cannot lead to the observed solar flare irradiation features in these meteorites. Goswami and Lal (1979) considered the difference between the exposure ages based on cosmogenic stable isotopes and radionuclides as the measure for pre-compaction irradiation duration. Such an approach is appropriate since the stable isotope age of a gas-rich meteorite will represent both the pre-compaction irradiation duration and the recent exposure duration of the meteorite as a small object in space, while the radionuclide exposure age will represent only the recent exposure duration in space.

As an alternative to the regolith scenario, Goswami and Lal (1979) have proposed that the solar flare and solar wind irradiation of individual components of gas-rich carbonaceous chondrites took place while they were residing in the surface or near surface regions of centimetre to metre size objects, prior to the formation of asteroidal size parent bodies. This must have happened in the very early stages of the evolution of the solar system. Lack of evidence for irradiation of individual grain in space requires that the pressure in the solar nebula was sufficient ( $>10^{-10}$ Atm.) to shield the grains from solar flare and solar wind particles until their incorporation into $\mathrm{cm}$ to metre size objects. Some of our recent studies (Goswami and Lal, unpublished) indicate that it is probable that some individual grains received direct solar flare irradiation, while being partially shielded by the gas pressure in the nebula itself. The centimetre to metre size bodies must coalesce to form larger size objects $(\geqslant \mathrm{km})$, within a short duration of $10^{4 \pm 1} \mathrm{yr}$, to effectively cut-off further solar flare and galactic cosmic ray irradiation, so that the constraint on the short pre-compaction irradiation duration can be satisfied. Collision among these large objects will finally lead to the formation of larger size parent bodies of $\mathrm{CI}$ and $\mathrm{CM}$ chondrites, which will thus form out of an irradiated swarm of objects, and shall contain 'irradiated ' material throughout its entire volume. This feature can readily explain the high percentage of gas-rich meteorites among the $\mathrm{CI}$ and $\mathrm{CM}$ chondrites. While impact-induced regolithic activity will definitely be present in the parent bodies of these meteorites and may lead to excavation, fragmentation and redistribution of material, the solarwind and solar flare records seen in individual components from the gas-rich members in these groups were essentially those imprinted prior to the formation of the parent bodies.

In view of the above development, a re-evaluation of our present understanding of the irradiation features seen in gas-rich meteorites as due to their exposure in asteroidal regoliths is becoming necessary. Work is currently in progress (Goswami and Nishiizumi, unpublished) to accurately determine the pre-compaction irradiation durations of gas-rich meteorites belonging to other chondritic and achondritic groups, based on measurements of noble gas and radionuclide exposure ages (on aliquots of the same sample). This approach, however, is feasible only in the case of meteo- 
rites with low exposure ages (e.g. H chondrites) since for meteorites with recent space exposure age exceeding $10 \mathrm{~m}$.y., the radionuclides $\left({ }^{26} \mathrm{Al},{ }^{53} \mathrm{Mn}\right)$ generally used for exposure age determination suffer from saturation effect. Additional experiments to determine the possibility of very different exposure histories of the irradiated and un-irradiated components in gas-rich meteorites are also necessary to get insight into the finer details of the exposure history of these meteorites. Future work along these directions could be expected to give us valuable information on the accretionary and evolutionary history of small objects in the early solar system.

\section{References}

Anders E 1975 Icarus 24363

Anders E 1978 in Asteroids: an exploration assessment, NASA Conf. Publ. 2053, p. 57

Arnold J R, Honda M and Lal D 1961 J. Geophys. Res. 663519

Arnold J R 1975 Proc. Lunar Sci. Conf. 6th (New York: Pergamon Press) p. 57

Bagolia C, Bhandari N, Sinha N, Goswami J N, Lal D, Lorin J C and Pellas P 1980 Bull. Astr. Soc. Czech. 3151

Bagolia C, Doshi N, Gupta S K, Kumar S, Lal D and Trivedi J R 1977 Nucl. Track Detection 183

Bagolia C, Lal D, Sinha N and Sears D 1978 Nucl. Track Detection 229

Baldwin B and Sheaffer Y 1971 J. Geophys. Res. 764653

Bhandari N 1981 Proc. Indian Acad. Sci. (Earth Planet. Sci.) 90359

Bhandari N, Lal D, Rajan R S, Arnold J R, Marti K and Moore C B 1980 Nucl. Tracks 4213

Bhandari N and Potdar M B 1981 Earth Planet. Sci. Lett. (in press)

Bhattacharya S K, Goswami J N and Lal D 1973 J. Geophys. Res. 788356

Bhattacharya S K, Imamura M, Sinha N and Bhandari N 1980 Earth Planet. Sci. Lett. 5145

Bull R K and Durrani S A 1976 Earth Planet. Sci. Lett. 3235

Cantelaube Y, Pellas P, Nordemann D and Tobailem J 1969 in Meteorite research, ed. P M Millman (Holland: D Reidel) p. 705

Crabb J and Schultz L 1981 Geochim. Cosmochim. Acta (in press)

Crozaz G 1980 in The ancient sun (New York: Pergamon Press) p. 331

Eberhardt P, Eugster O, Geiss J and Marti K 1966 Z. Natuurforsch. A21 414

Fleischer R L, Price P B, Walker R M, Maurette M and Morgan 1967 J. Geophys. Res. 72355

Gault D E, Horz F, Brownlee D E and Hartung J B 1974 Proc. Lunar Sci. Conf. 5 th (New York: Pergamon Press) p. 2365

Goswami J N, Lal D, Rao M N, Sinha N and Venkatesan T R 1978 Meteoritics 13481

Goswami J N and Lal D 1979 Icarus 40510

Goswami J N, LaI D and Macdougall J D 1980 in The ancient sun (New York: Pergamon Press) p. 347

Gupta S K and Lal D 1978 Nucl. Track Detection 237

Housen K R, Wilkening L, Chapman C R and Greenberg R 1979 Icarus 39317

Imamura M, Nishiizumi K and Honda M 1979 Mem. Natl. Inst. Polar Res. 15227

Jha $R$ and Lal D 1981 (in preparation)

Lal D 1972 Space Sci. Rev. 143

Lal D, Lorin J C, Pellas P, Rajan R S and Tamhane A S 1969 in Meteorite research, ed. P Millman (Holland: D Reidel) p. 275

Lal D and Rajan R S 1969 Nature (London) 223269

Langevin Y and Arnold J R 1977 Annu. Rev. Earth Planet. Sci. 5449

Langevin Y and Maurette M 1980 in Lunar Planet. Sci. 11 (Houston: Lunar Sci. Inst.) p. 602

Lorin J C and Pellas P 1975 Meteoritics 10445

Macdougall J D and Kothari B K 1976 Earth Planet. Sci. Lett. 3336

McCrosky R E 1968 in Research in space science, Smithsonian Astrophys. Observatory Sp. Report No. 280, p. 1

Cpik E J 1958 Physics of meteor flight in the atmosphere (New York: Interscience) 
Pellas P 1972 in Proc. Noble Symp. 21: From plasma to planets (New York: John Wiley) p. 65

Pellas P, Poupeau G, Lorin J C, Reeves H, Audoue J 1969 Nature (London) 223272

Poupeau G and Berdot J L 1972 Earth Planet. Sci. Lett. 14381

Poupeau G, Kirsten T, Steinbrunn F and Storzer D 1974 Earth Planet. Sci. Lett. 24227

Price P B, Rajan R S and Tamhane A S 1967 J. Geophys. Res. 721377

Price P B, Hucheon I D, Braddy D and Macdougall J D 1975 Proc. Lunar Sci. Conf. 6th (New York Pergamon Press) p. 3449

Rajan R S 1974 Geachim. Cosmochim. Acta 38777

Rajan R S, Huneke J C, Smith S P and Wasserburg G J 1979 Geochim. Cosmochim. Acta 43957 Reedy R C, Arnold J R and Lal D 1981 Preprint

Revelle D O 1978 in Carnegie Institution, Department of Terrestrial Magnetism, Washington D.C Annual Report, p. 475

Schultz L and Signer P 1977 Earth Planet. Sci. Lett. 36363

Singhvi A, Sengupta D and Bhandari N 1981 (in preparation)

Suess H E, Wanke H and Wlotzka F 1964 Geochim. Cosmochim. Acta 28595

Turner G 1979 Proc. Lunar Planet Sci. Conf. 10th (New York: Pergamon Press) p. 1917

Walker R M 1975 Annu. Rev. Earth Planet. Sci. 399

Wanke H 1966 Fortschr. Chem. Forsch. 7322

Wetherill G W 1980 in Carnegie Institution, Department of Terrestrial Magnetism, Washington D.C. Annual Report, p. 546

Wilkening I 1971 in Particle track studies and the origin of gas-rich meteorites (Tempe: Arizona State Univ.) p. 1 